\title{
Distortion of power law blinking with binning and thresholding
}

Nicole Amecke, André Heber, and Frank Cichos

Citation: The Journal of Chemical Physics 140, 114306 (2014); doi: 10.1063/1.4868252

View online: https://doi.org/10.1063/1.4868252

View Table of Contents: http://aip.scitation.org/toc/jcp/140/11

Published by the American Institute of Physics

\section{Articles you may be interested in}

Nonexponential "blinking" kinetics of single CdSe quantum dots: A universal power law behavior The Journal of Chemical Physics 112, 3117 (2000); 10.1063/1.480896

"On"/"off" fluorescence intermittency of single semiconductor quantum dots

The Journal of Chemical Physics 115, 1028 (2001); 10.1063/1.1377883

Photothermal single particle microscopy using a single laser beam

Applied Physics Letters 105, 013511 (2014); 10.1063/1.4890104

Photonic Rutherford scattering: A classical and quantum mechanical analogy in ray and wave optics American Journal of Physics 81, 405 (2013); 10.1119/1.4798259

Beyond power laws: A new approach for analyzing single molecule photoluminescence intermittency The Journal of Chemical Physics 136, 184508 (2012); 10.1063/1.4717618

Back focal plane imaging spectroscopy of photonic crystals

Applied Physics Letters 101, 081904 (2012); 10.1063/1.4746251

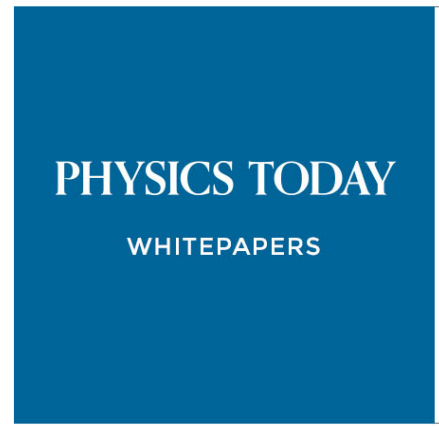

ADVANCED LIGHT CURE ADHESIVES

Take a closer look at what these environmentally friendly adhesive systems can do

\section{READ NOW}

PRESENTED BY Q. MASTERBOND" 


\title{
Distortion of power law blinking with binning and thresholding
}

\author{
Nicole Amecke, André Heber, and Frank Cichos ${ }^{a)}$ \\ Molecular Nanophotonics Group, Institute of Experimental Physics I, Universität Leipzig, Linnéstraße 5, \\ 04103 Leipzig, Germany
}

(Received 16 December 2013; accepted 27 February 2014; published online 18 March 2014)

\begin{abstract}
Fluorescence intermittency is a random switching between emitting (on) and non-emitting (off) periods found for many single chromophores such as semiconductor quantum dots and organic molecules. The statistics of the duration of on- and off-periods are commonly determined by thresholding the emission time trace of a single chromophore and appear to be power law distributed. Here we test with the help of simulations if the experimentally determined power law distributions can actually reflect the underlying statistics. We find that with the experimentally limited time resolution real power law statistics with exponents $\alpha_{\text {on/off }} \gtrsim 1.6$, especially if $\alpha_{\text {on }} \neq \alpha_{\text {off }}$ would not be observed as such in the experimental data after binning and thresholding. Instead, a power law appearance could simply be obtained from the continuous distribution of intermediate intensity levels. This challenges much of the obtained data and the models describing the so-called power law blinking. (C) 2014 AIP Publishing LLC. [http://dx.doi.org/10.1063/1.4868252]
\end{abstract}

\section{INTRODUCTION}

Uncovered by single particle microscopy, fluorescence intermittency of single emitters remains an equally puzzling as universal phenomenon. Not only semiconductor quantum dots (QDs), but also molecules, proteins, nanorods, or nanowires, exhibit random fluctuations between emitting on- and non-emitting off-states ${ }^{1,2}$ occurring on timescales ranging from microseconds to tens or hundreds of seconds. Particularly remarkable are the discovered statistics reflecting the probability of finding on- or off-periods of a certain duration. These dwell times in either state appear to follow an inverse power law distribution

$$
p(t) \propto t^{-\alpha} \quad(1<\alpha<2)
$$

with an exponential cut-off $e^{-t / \tau_{\text {cut-off }}}$ for the on-states. The off-state statistics are found to be surprisingly robust against variations of temperature or excitation intensity. This robustness suggests a very fundamental process ${ }^{3-5}$ to be active. On the contrary, the on-state statistics with their cut-off are sensitive to temperature, excitation, and particle morphology. ${ }^{6,7}$ This sensitivity renders the $o n$-states particularly interesting to a further study of the fundamentals of the blinking phenomenon. Recently it could be shown that at least two blinking mechanisms exist. One mechanism was ascribed to charge ejection with subsequent Auger quenching with an exponential distribution of dwell times. ${ }^{8}$ The power law distribution, however, arises from a different mechanism, ${ }^{3,8}$ whose origin remains under discussion. ${ }^{9}$ Both mechanisms can be inhibited with gradient and/or thick shells emphasizing the importance of the QD interface and surface. A true understanding of the mechanism leading to a power law distribution nevertheless remains to be found.

\footnotetext{
a)cichos@physik.uni-leipzig.de
}

Such power law distributed dwell times are rather unexpected for the transition between two well-defined states, which would be expected to show exponential distributions. Thus, several models were developed seeking the origin of blinking. ${ }^{1,2,6,10-12}$ These models largely address the power law statistics as their central feature, relying on the fact that the experimental analysis truly reflects the physical reality. While in the case of fluorescence intermittency a number of different methods exists to analyze the experimental photon streams, ${ }^{11,13-18}$ the distinct separation of on- and off-state is almost exclusively performed with the thresholding method. Here an intensity time-trace is recorded and a threshold intensity chosen to define on-states (above the threshold) and off-states (below the threshold). The occurrence of on- and off- intervals of different length is then displayed in log-log histograms. The time resolution of these time-traces is inherently bound to their bin-time $t_{\text {bin }}$. In the past years it has been more and more realized that this bin-time and the decision on a threshold strongly define the resulting histograms especially for data with low signal to noise ratios. ${ }^{19-21}$ It was found not only problematic in the analysis of quantum dots or rods ${ }^{20}$ but for any single nano-object. ${ }^{19}$ In the study of enzymatic reactions ${ }^{21}$ it was even suggested that the often proposed dynamic disorder extracted via thresholding might only be an artifact of analysis.

As we present here, also for semiconductor nanocrystals the appearance of certain power law distributions will be strongly biased by analysis artifacts. Typical experimental bin-times lie in the range of $1-100 \mathrm{~ms}$. The onset of the observed power law blinking is believed to reach down to much shorter times. This onset is often referred to as the critical time $t_{c}$. It is the shortest dwell time in either state with the same power law probability as observed for long times. In electron diffusion models it marks a change of blinking statistics to lower exponents. ${ }^{12}$ Using power spectra evidence is reported that this critical time $t_{c}$ might be close to the 
typical bin width $\left(t_{c} \approx 10 \mathrm{~ms}\right) .{ }^{14}$ However, based on correlation functions ${ }^{11,15}$ and fluorescence decays ${ }^{22}$ the same power law statistics are observed to reach down to the microsecond scale or even beyond. This limited time resolution would strongly distort the observed statistics of true power law blinking, if $t_{c} \nsucc t_{\text {bin. }}$. By simulating time-traces with defined blinking parameters, we show that for many experimentally determined exponents pure power-law statistics would hardly be observed in both states. This especially applies to the on-state, when the on-state cut-off, experimental noise, and typical thresholds are considered. The distortion is found for all power law exponents except for the special case of low and equal exponents $\left(\alpha_{\text {on }}=\alpha_{\text {off }} \lesssim 1.6\right)$, which is the one most intensively tested against simulations. $.^{20,23,24} \mathrm{Sim}-$ ulations with higher exponents showed similar distortions as observed here..$^{25}$ Thus, most of the currently observed statistics most likely do not reflect real statistics. This stresses the importance of $t_{c}$ with respect to $t_{\mathrm{bin}}$ and even poses the question if real statistics really follow power law distributions for both states. We find that the observed statistics can instead be highly influenced by the Poisson noise of intermediate states, which would then also appear power law distributed. Based on these findings most measurements and determined dependencies should be revisited. Also blinking models might need to be adjusted.

\section{SIMULATION}

Time-traces are simulated based on power law distributed random numbers $t_{\text {on/off }}$ for on- and off-states with given power law exponents $\alpha_{\text {on/off. }}$ Those are generated from equally distributed random numbers $(r \in[0,1])$ and then transformed by $t_{\text {on } / \text { off }}=r^{1 /\left(1-\alpha_{\text {on/off }}\right)}{ }^{26}$ yielding $t_{\text {on/off }}>1$. On- and off- times are thus obtained in reference to a minimum time, the critical time $t_{c}$ for the onset of blinking, which is set to $t_{c}=1$ per definition. For power law distributions with cut-off, $\tau_{\text {cut-off }}$, the resulting number $t_{\text {on }}$ is only accepted with probability $e^{-t_{\text {on }} / \tau_{\text {cut }- \text { off }} \text {. We restrict the random numbers to } 2 \times 10^{4} t_{\text {bin }} \text { as a }}$ single measurement of one quantum dot is usually not longer than $10^{4} t_{\mathrm{bin}}$. For on- and off-states a random number from the distribution is drawn alternately. The on-state intensity is set to 1 , the off-state intensity to 0 . Now a time-trace of resolution $t_{\text {bin }} \geq t_{\mathrm{c}}$ is constructed where the intensity of each bin is given by the fraction of time spent in the on-state. If we take $1 \mu \mathrm{s}$ as a lower bound for the start of power law blinking, ${ }^{15}$ typical bin-times ( 1 to $100 \mathrm{~ms}$ ) are three to five or even more orders of magnitude larger. Thus, we choose $t_{\text {bin }}=10^{k} t_{\mathrm{c}}$, testing $k=0-5$, including values of $t_{c} \approx t_{\text {bin }} \cdot{ }^{14}$ Like in experiments, the dwell times $t_{\text {on/off }}$ in either state are found with a threshold and are separately displayed in histograms for on- and off-states. Those are logarithmically binned, increasing the bin width to $2^{n} t_{\text {bin }}, n=0,1,2 \ldots$ and presented on logarithmic axes. An observed straight line would then represent an observed power law and its slope the exponent.

\section{RESULTS AND DISCUSSION}

\section{A. Influences of binning and threshold}

The influence of a decreasing time resolution is first investigated for equal exponents of on- and off-state. As we will show, it is strongly coupled to the chosen threshold.

A typical simulated time-trace and its intensity histogram for $\alpha_{\text {on }}=\alpha_{\text {off }}=1.8$ and $t_{\text {bin }}=10^{4} t_{\mathrm{c}}$ is depicted in Fig. 1 . The intensity changes between 1 (on) and 0 (off). Also intermediate intensities are found due to unresolved blinking events, which we refer to as "unresolved bins." Most are found at half the on-state intensity $\left(0.5 I / I_{\text {on }}\right)$. The amount of unresolved bins quickly increases with bin-time until it saturates. Fig. 2(a) shows intensity histograms for time-traces for different bin-times and $\alpha_{\text {off }}=\alpha_{\text {on }}=1.8$. The asymptotic shape of the histogram for long bin-times is given by the Lamperti function (see Eq. (S4) of the supplementary material ${ }^{27}$ and Refs. 28 and 29).

Now different thresholds can be chosen to obtain the distributions of on- and off-events. As the exponents are the same for on- and off-state, a threshold at 0.5 yields identical histograms for both distributions. Furthermore, identical distributions will be observed for off-states at a low threshold (e.g., 0.1) and on-states at the corresponding high threshold (e.g., 0.9) and vice versa. Thus, only the off-state distributions
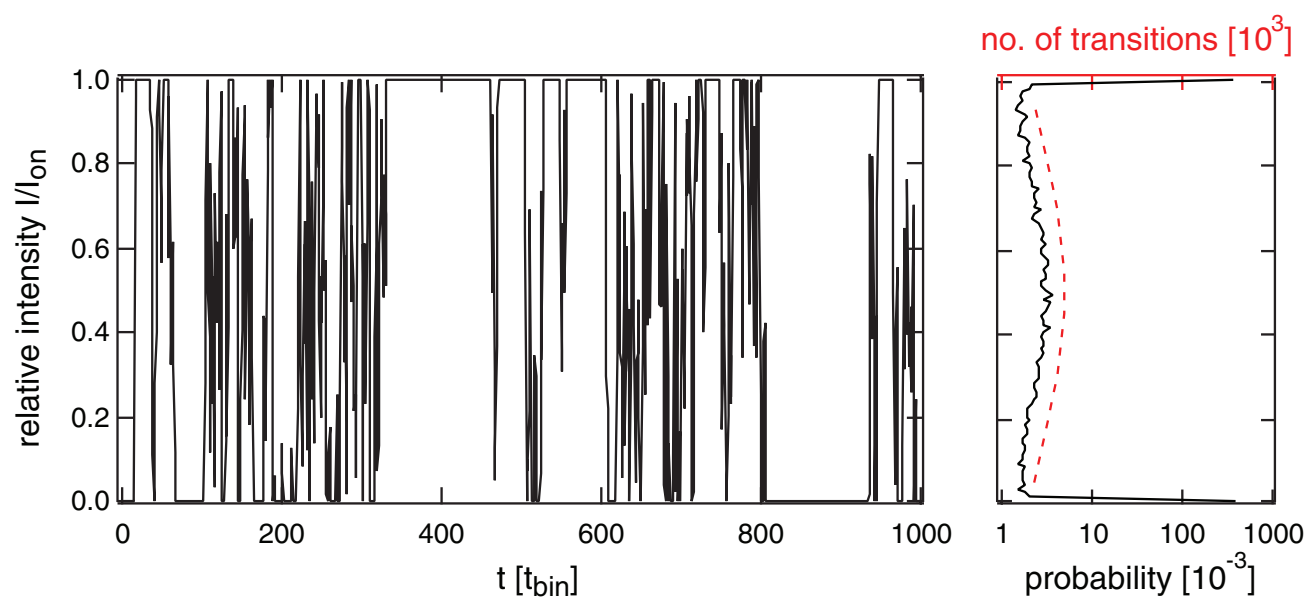

FIG. 1. Left: Extract of a simulated time-trace for $\alpha_{\text {off }}=\alpha_{\text {on }}=1.8$ and $t_{\text {bin }}=10^{4} t_{\mathrm{c}}$. Right: Probability histogram over the simulated intensities (black solid line, bottom axis) and number of detected events (red dashed line, top axis), both on logarithmic scales. Intensity is given in on-state fraction. 

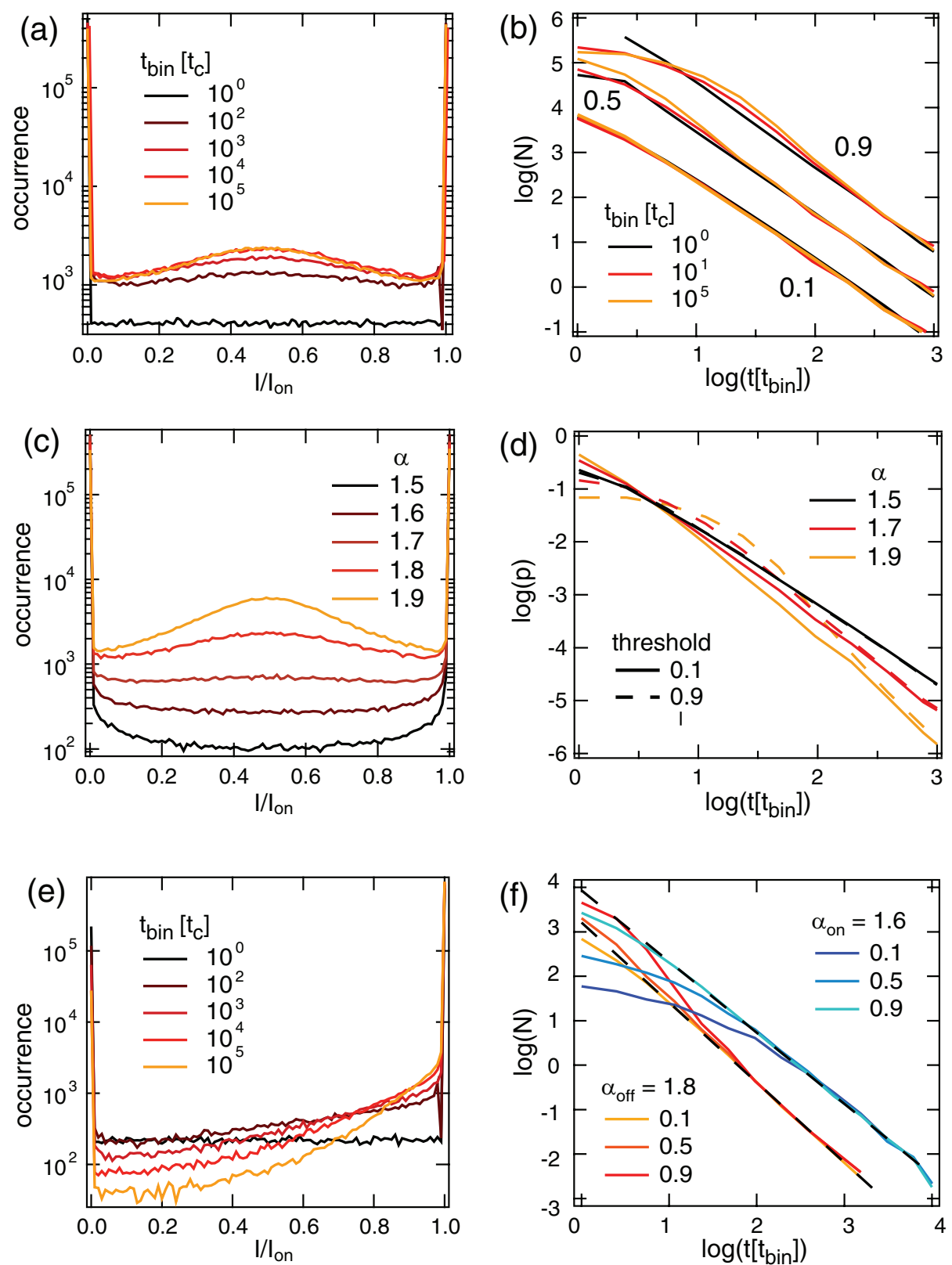

FIG. 2. (a) Intensity histograms of simulated time-traces with $\alpha_{\text {off }}=\alpha_{\text {on }}=1.8$ and different $t_{\text {bin }}$. (b) Corresponding occurrence histograms for different thresholds and $t_{\text {bin }}$. Thresholds 0.5 and 0.9 are offset for clarity. (c) Intensity histograms of simulated time-traces with $t_{\text {bin }}=10^{4} t_{\mathrm{c}}$ and different $\alpha_{\text {off }}=\alpha_{\text {on }}$. The hump at $I / I_{0}=0.5$ increases with increasing exponent. (d) Probability histograms of off-states for thresholds 0.1 and 0.9 for different exponents $\alpha_{\text {off }}=\alpha_{\text {on }}$ and $t_{\text {bin }}=10^{4} t_{\mathrm{c}}$. With increasing exponent deviations between the two thresholds increase. (e) Intensity histograms of simulated time-traces with $\alpha_{\text {off }}=1.8$ and $\alpha_{\text {on }}=1.6$ with increasing $t_{\text {bin }}$. The amount of unresolved bins close to the on-state increases. (f) Corresponding off- and on-state occurrence histograms for $t_{\mathrm{bin}}=10^{4} t_{\mathrm{c}}$ and different thresholds. At short times strong deviations from the input power law are found especially for the on-state with the lower exponent.

are discussed in the following. For selected bin-times corresponding off-state histograms are displayed for three thresholds $0.1,0.5$, and 0.9 in Fig. 2(b). Obviously, only for bin-times close to the onset of blinking $t_{c}$ (black line, excluding the first bin), the observed histograms are independent of the threshold and reflect the input power law. With increasing bin-time also a threshold dependence develops. This dependence is already substantial for bin-times only one order of magnitude longer than $t_{\mathrm{c}}$ (Fig. 2(b), red line). Like the number of observed unresolved bins, it saturates for long bin-times.

This threshold dependence can be described as follows. For low thresholds close to the off-state the input power law is observed independent from bin-time. With increasing threshold the distribution of short off-events is increasingly distorted. Only at long events $>100 t_{\text {bin }}$ the input power law is recovered for all thresholds. The distortion, thus, increases with the number of unresolved bins mistakenly counted as off-events. At low thresholds this number is close to zero. With increasing threshold, fluctuations between unresolved bins first introduce more apparent short states. At even higher thresholds consecutive off-states are combined to intermediate states. This leads to a bending of the histogram for short times. The number of unresolved bins at a given intensity also follows closely the number of transitions that are detected 
between on- and off-state when this intensity is chosen for the threshold (red dashed line in Fig. 1). The maximum of transitions at medium thresholds only feigns better accuracy. Additional detected events, however, only distort the distribution.

Extracting the input exponents with a fit to the observed histograms is clearly difficult. Histograms at high thresholds do not resemble a power law at all. Fits to histograms at lower thresholds will surely yield exponents higher than the input ones, when all short events are included. Thus, the given experimental condition of $t_{\mathrm{c}} \ll t_{\text {bin }}$ already introduces considerable inaccuracies in the detected on- and off-state statistics.

\section{B. Dependence on power law exponent}

The strong distortion by the limited time resolution has not been found before, ${ }^{20,23,24}$ even though similar simulations were applied. The reason for this is that only low exponents $\leq 1.7$ were considered. For lower exponents the fraction of time spent in events of length $<t_{\text {bin }}$ decreases (see Fig. S1b of the supplementary material ${ }^{27}$ ). This fraction is closely related to the number of unresolved bins, which are distorting the histograms. The influence of different power law exponents is demonstrated in Figs. 2(c) and 2(d). Fig. 2(c) shows the intensity histograms for different exponents at $t_{\text {bin }}=10^{4} t_{\mathrm{c}}$. Those are again asymptotically described by the Lamperti equation (Eq. (S4) of the supplementary material ${ }^{27}$ ) for long bin-times. Corresponding off-state probability histograms for different $\alpha$ and thresholds 0.1 and 0.9 are shown in Fig. 2(d). For $\alpha=1.5$ no threshold dependence is observed. Only the first two time bins are slightly lower than expected for the input power law. For higher exponents, however, the threshold dependence for short events grows increasingly important. Thus, the extraction of higher power law exponents should be treated with care. A threshold dependence should be observed, similar to the one described here. If this is not the case, the determined exponents are not related to an underlying power law.

\section{Different exponents for on- and off-state}

Several experiments suggest that on- and off-exponents are equal. ${ }^{6,23}$ However most experiments extract independent exponents, which can differ considerably. For independent exponents the threshold method has also not been tested so far. This is investigated in the following. Representatively, the case of $\alpha_{\text {on }}<\alpha_{\text {off }}$ is examined. A lower on-time exponent implies that long events are more likely and short events less likely for the on-state as compared to the off-state. Thus more time will be spent in the on-state. Intensity histograms for different binning but a lower on-state exponent $\left(\alpha_{\text {on }}=1.6\right)$ are depicted in Fig. 2(e). The amount of unresolved bins close and equal to 1 increases with bin-time. The shape of the histograms is analytically described in Ref. 28 (Eq. (7)). For long bin-times, the QD will predominantly stay in the on-state and blinking will be suppressed. $O n$ - and off- state histograms for $t_{\text {bin }}=10^{4} t_{\mathrm{c}}$ and thresholds $0.1,0.5$, and 0.9 are displayed in Fig. 2(f). Again, a strong threshold dependence of the statistics of short events is observed for both distributions, while the statistics of long events are unperturbed. The effect is stronger for the on-state with the lower exponent. Only for thresholds close to the corresponding state, the input power law is observed throughout the whole histogram. The more unresolved bins are included, the stronger the distortion. As most unresolved bins are close to the on-state, the on-state distribution is quickly destroyed when lowering the threshold. Short on-events are no longer discriminated and the histogram flattens.

On the contrary, the off-state distribution is almost threshold independent for low thresholds. Only at very high thresholds, switching between unresolved bins leads to an increasing amount of apparent short off-times. The deviation from the input power laws shifts to longer times with longer bintimes, higher exponents, and higher difference of $\alpha_{\text {on }}$ and $\alpha_{\text {off }}$. In typical experiments the exponent 1.6 for the on-times in Fig. 2(f) would not be determined correctly. A much lower exponent would be fitted to the initial histogram, especially when long times are missing due to a cut-off (see below). Such an even lower exponent however would lead to a completely different histogram. Thus, even more than the extraction of high power law exponents, fitting results yielding differences in on- and off- state exponent should be treated with care. Most of those cannot correctly describe underlying power law distributions.

\section{Exponential on-state cut-off and Poisson noise}

Two additional difficulties have to be met in experiments: the exponential on-state cut-off and Poisson noise. An onstate cut-off $\tau_{\text {cut-off }}$ much longer than the bin-time only eliminates long on-times. The distribution of off-times and short on-times is unaffected. The effect of different $\tau_{\text {cut-off }}$ on the on-time statistics are displayed for high (dashed) and low (solid) thresholds in Fig. 3(a). The histograms show that for $\tau_{\text {cut-off }} \lesssim 100 t_{\text {bin }}$ the observed cut-off time depends considerably on the chosen threshold. It shifts to longer times with decreasing thresholds. Also very short $\tau_{\text {cut-off }}$ of only $2 t_{\text {bin }}$ can show a pure exponential distribution with much higher decay constant ${ }^{19}\left(16 t_{\text {bin }}\right)$ at threshold 0.1 (see Fig. 3(b), black line). This can easily be misinterpreted as the actual $\tau_{\text {cut-off }}$. Such short cut-offs $\lesssim t_{\text {bin }}$, rarely lasting over more than one bin, also decrease the occurrence of the maximum intensity in a time-trace. This means, the real on-state intensity can no longer be identified. Without this knowledge the choice of thresholds at specific on-state fractions and thus a comparison between measurements is no longer possible. Even longer cut-offs often prevent the determination of a possible on-state power law, as they eliminate long on-times, which are the only ones not distorted by unresolved bins. The determination of off-state statistics, however, benefits in two ways by a cut-off. First, the increased number of events at the same measurement time leads to better statistics also in the unperturbed long events. And second, the low probability of on-events at maximum intensity usually leads to lower thresholds, most suitable for detection of off-state statistics.

For Poisson noise it has been shown ${ }^{19,20}$ that the threshold must be chosen outside of the noise of either state. To 

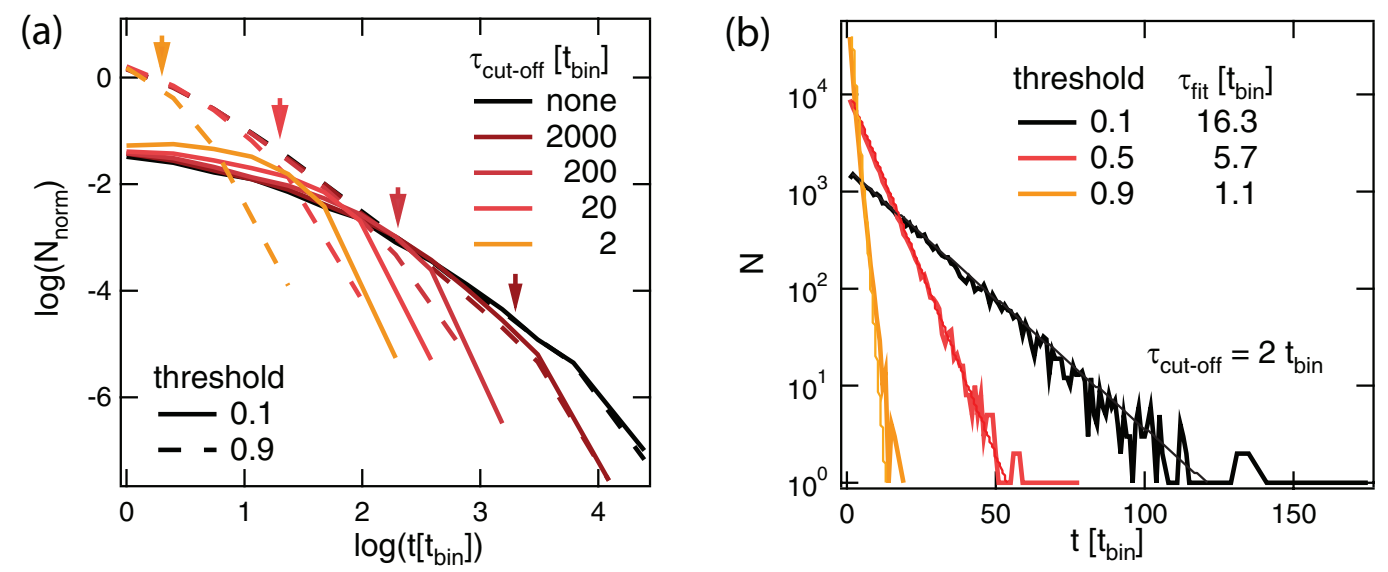

FIG. 3. (a) On-state occurrence histograms for different $\tau_{\text {cut-off }}>t_{\text {bin }}$ (colors) and different thresholds (line style) with $\alpha_{\text {off }}=1.8, \alpha_{\text {on }}=1.6$, and $t_{\text {bin }}=10^{4} t_{\mathrm{c}}$. The histograms are normalized $\left(N_{\text {norm }}\right)$ for each $\tau_{\text {cut-off }}$ to the number of detected events at threshold 0.1 . Arrows mark the corresponding $\tau_{\text {cut-off. }}$ (b) $O n$-state occurrence histograms for $\tau_{\text {cut-off }}=2 t_{\text {bin }}$ at different thresholds. Results of single exponential fits $\left(\tau_{\text {fit }}\right)$ are indicated.

examine this closer, Poisson noise corresponding to a typical signal to background ratio 100:3 (10 ms binning, $300 \mathrm{cps}$ background ( 3 cts off-state), $10 \mathrm{kcps}$ signal (103 cts on-state)) is added to the time-trace of Figs. 2(e) and 2(f). The different thresholds that are investigated are indicated in the histogram in Fig. 4(a) and referred to by their on-state fraction.
As shown in Fig. 4(b), noise of unresolved bins does not influence the histograms. All prior effects thus remain unaffected also for data including Poisson noise, when thresholds are placed sufficiently outside of the noise. However, strong distortions are found, when the threshold lies within the noise of either state, like shown for the on-state noise in Figs. 4(c)
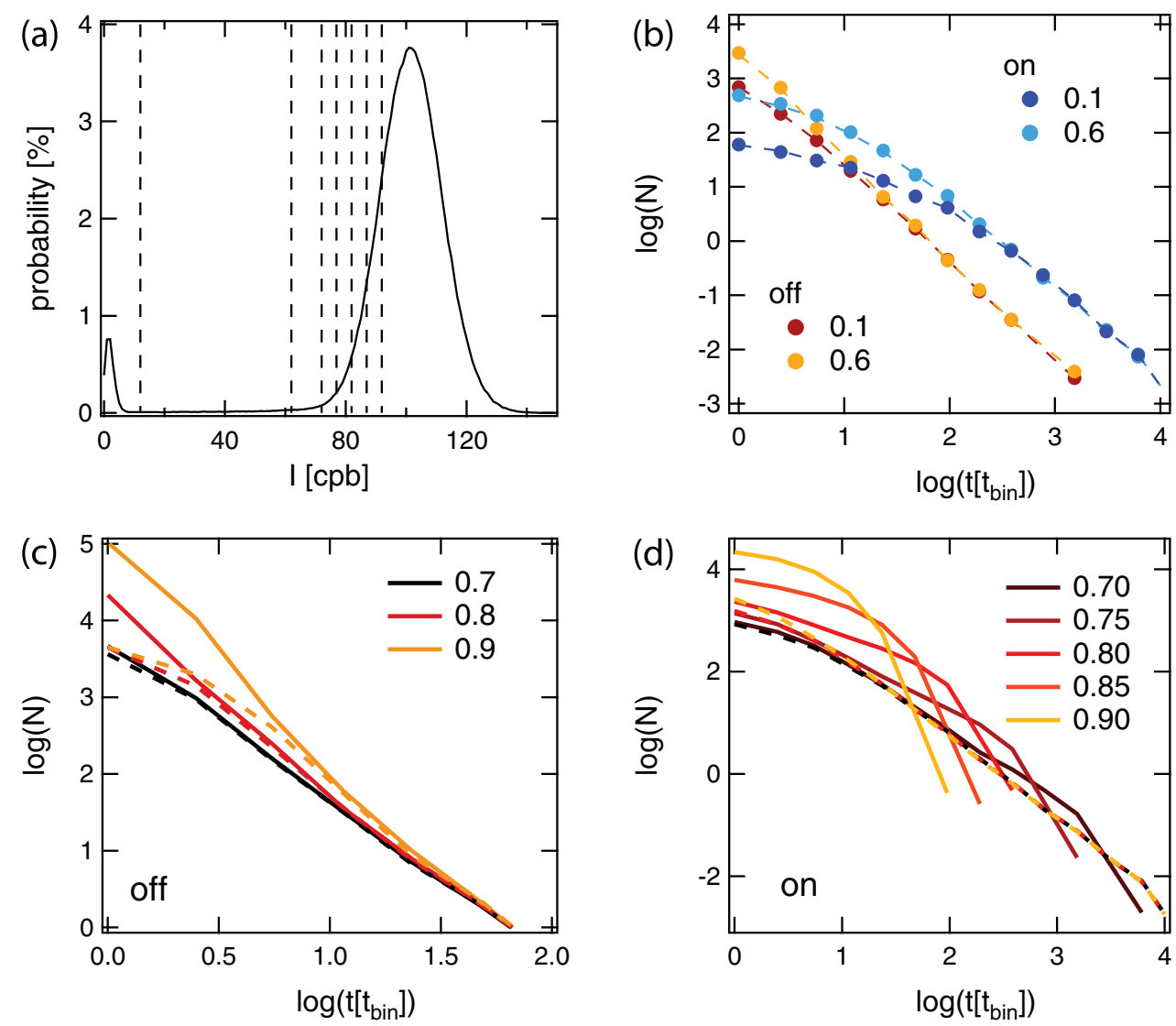

FIG. 4. (a) Intensity histogram of the time-trace to Fig. 2(f) with $\alpha_{\text {off }}=1.8, \alpha_{\mathrm{on}}=1.6$ and $t_{\text {bin }}=10^{4} t_{\mathrm{c}}$ with Poisson noise. An on-state signal of 100 photons per bin is assumed and 3 background photons per bin are added. The investigated thresholds of b)-d) are marked as dashed lines. (b) Off- and on-state histograms for two thresholds outside the noise, showing no influence of the noise. (c) Off-state and (d) on-state histograms for thresholds from 0.7 to 0.9 . When the threshold reaches the Poisson noise of the on-state, a cut-off is introduced in the on-state histogram, which shifts to shorter times with higher threshold. The off-state histogram shows more short states with increasing threshold. Dashed lines in b-d) are without noise. 

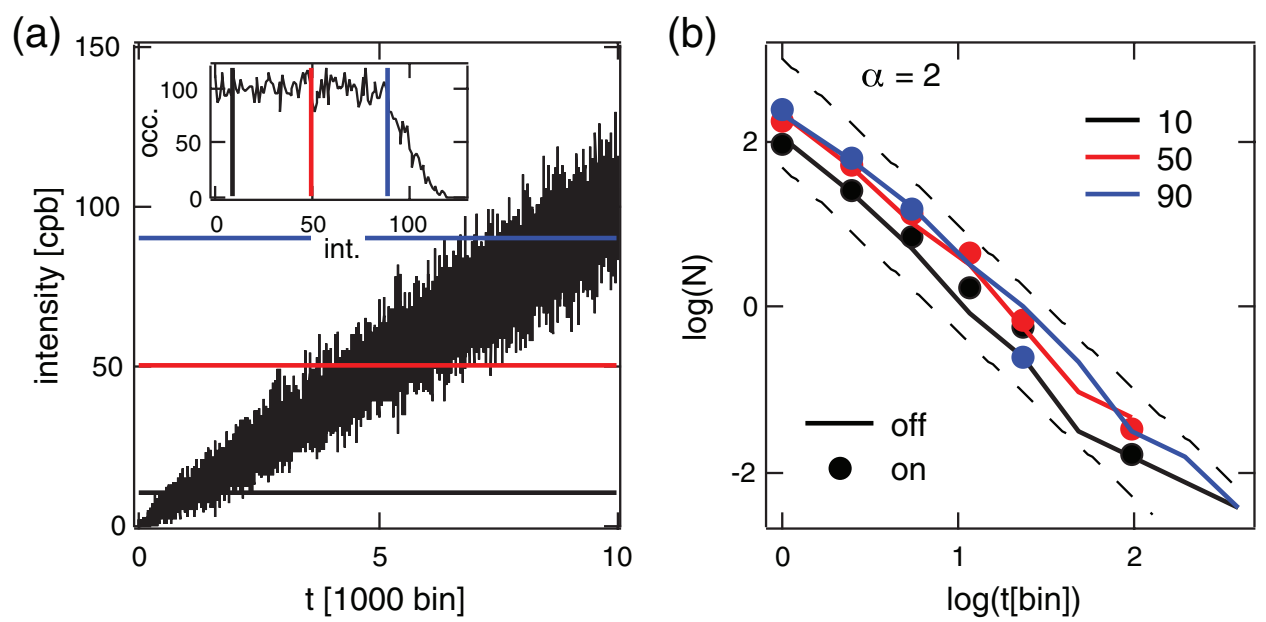

FIG. 5. (a) Linearly increasing intensity with Poisson noise given in counts per bin and corresponding histogram in the inset. The three investigated thresholds are marked in black, red, and blue. (b) Obtained power law log-log histogram for on- and off- events after the threshold analysis on the time-trace in (a). Dashed lines mark slopes of $\alpha=2$.

(off-state) and 4(d) (on-state). The off-states in Fig. 4(c) show an increasing amount of short times in the histogram, while long times are not affected. The histogram of the on-states in Fig. 4(d) immediately shows a cut-off for long durations, which shifts to shorter times, when the threshold moves deeper into the noise. For pure Poisson noise the time of the induced cut-off $\tau_{\text {on,off }}$ at a given threshold $T$ can be estimated from the count rates $I_{\text {on }}$ and $I_{\text {off }}$ of on- and off-state according to (see the supplementary material ${ }^{27}$ )

$$
\tau_{\mathrm{on}}\left[t_{\mathrm{bin}}\right]=\frac{\Gamma(T)}{\Gamma\left(T, I_{\mathrm{on}}\right)} \quad \tau_{\mathrm{off}}\left[t_{\mathrm{bin}}\right]=\left[1-\frac{\Gamma\left(T, I_{\mathrm{off}}\right)}{\Gamma(T)}\right]^{-1} .
$$

The missing long times will be found at intermediate time, leading to a small hump in the histogram. Similar humps have been observed in on-state distributions. ${ }^{30}$ They however follow a different threshold dependence, and thus are likely not simply noise induced. As has often been stated before, it is thus crucially important to choose a threshold outside the noise. This unfortunately prevents the best choices of thresholds, closest to the corresponding states. Only those would lead to the least distorted dwell time histograms by unresolved bins. It especially limits adequate $o n$-state thresholds due to its higher noise. $O n$-state cut-off and noise often render an identification of a presumed power law for the $o n$-state impossible. The inevitably low threshold distorts the short events, while the cut-off removes the unperturbed long events.

\section{E. Real intermediate intensity levels}

While this paper focuses on the difficulties arising from the threshold method even for simple two-state blinking, experiments show a more complicated system. Additional discrete intermediate intensity levels have been found ${ }^{31,32}$ as well as indications of a continuous distribution of intensity states between maximum and background levels. ${ }^{17,33}$ They can be distinguished from mere unresolved blinking events by their different excited state decay time. Not much is known about dwell times and intensity changes in this continuous distribution. Nevertheless, they will contribute to the statistics determined by thresholding. To give an example of possible effects of intermediate intensity levels, a simple time-trace with linearly increasing intensity is investigated. It is depicted in Fig. 5(a). The histogram in the inset depicts the corresponding continuous distribution of intensity. When the threshold method is applied to this time-trace at the indicated thresholds, an inverse power law is obtained as well. Its exponent lies only slightly below 2 as indicated by the dashed lines representing $\alpha=2$. Any combination of threshold and count rate induce a different exponential distribution with time constants given by Eq. (2). A continuous distribution of count rates leads to the power law like dwell time histogram as observed in Fig. 5. Before interpreting power laws to the determined statistics, the influence from intermediate intensity levels thus needs to be considered. Their shot noise close to the threshold may lead to power law like statistics as well. Models to intermediate level hopping are thus in demand. As the chosen example demonstrates, also uncorrected drifts of QDs, fluctuations in excitation intensity, and bleaching will contribute to a power law appearance of the detected statistics.

\section{F. Implications for experiments}

In experiments usually the same threshold is chosen for on- and off-state distributions, often as the off-state intensity plus several times its standard deviation. ${ }^{20}$ As this threshold generally lies close to the off-state, the corresponding off-state distribution can well be determined. An observed power law can thus be trusted to reflect underlying statistics if several orders of magnitude are found. One should however make sure that no drifts or intensity fluctuations are present and there is a low occurrence of intermediate intensity values (unresolved or real) close to the threshold value.

However, if not $t_{\text {bin }} \sim t_{c}$, with such a low threshold even pure power law switching would not lead to an on-state power law distribution for high $\left(\alpha_{\text {off, on }}>1.6\right)$ and/or differing exponents of $o n$ - and off-state, especially not for $\alpha_{\text {on }}<\alpha_{\text {off }}$. Also 
for $\alpha_{\text {on }}>\alpha_{\text {off }}$ a strong threshold dependence for the off-state distribution should be, but is generally not, observed in experiments. The mentioned exponents, however, are fit to onstate distributions..$^{25,29,34,35}$ Hence, the so-determined power laws for the $o n$-states are not reflecting real dwell time statistics. The true statistics might not even follow a power law at all, at least not the one that was extracted. Convincing onstate power laws have hardly been found over several orders of magnitude. Summing over many QDs can quickly be misleading. Different $\tau_{\text {cut-off }}$ or different QD intensity with thresholds in the noise can similarly resemble a power law as that in Fig. 5. For single QDs effects of drifts, intensity fluctuations and intermediate levels need to be excluded. For instance, a high occurrence of intensity values close to the threshold in the intensity histogram is suspicious. If a power law is still observed a comparison to simulations as here can help to verify the assignment. This always requires an assumption to the onset of power law blinking $t_{c}$, which again stresses its relevance. In most cases the only conclusion could be an onset of power law blinking very close to the bin time $\left(t_{c} \sim t_{\text {bin }}\right)$. This would as well be interesting for the assignment of the physical process. A fixed shortest dwell time $t_{c}$ as used in the simulations will certainly not reflect the real situation. The critical time $t_{c}$ could only indicate a change in blinking statistics as in electron diffusion models. ${ }^{12}$ It can also differ for on- and offstate and depend on experimental parameters like the excitation rate. All physical models rely on a shortest time range for which the long time power law statistics are still valid. More investigations of the short time blinking behaviour are thus needed to determine $t_{c}$. Relevant timescales might only be accessible with correlation functions, power spectra, and fluorescence lifetime decays. Carefully drawn conclusions need to be scrutinized with regard to observable implications to blinking time-traces as described here.

The appearance of a cut-off limits the determination of potential power law kinetics at long times but also introduces another parameter for the $o n$-state characterization. While this limit to the length of on-times is observed, the extraction of reliably values for the time $\tau_{\text {cut-off }}$ is limited. Only for values $10 t_{\text {bin }}<\tau_{\text {cut-off }}<0.1 t_{\text {tot }}$ and $\tau_{\text {cut-off }}<\tau_{\text {on }}$ (Eq. (2)) a meaningful extraction is possible. Thresholds in the noise will always introduce artificial cut-offs at $\tau_{\text {on }}$ (see Fig. 4(d)). A total measurement time $t_{\text {tot }}<10 \tau_{\text {cut-off }}$ is too short to allow signif-

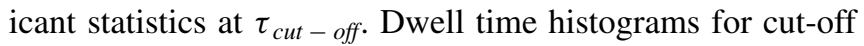
times shorter than $10 t_{\text {bin }}$ are distorted by the limited time resolution (see Fig. 3(b) as well as Refs. 19 and 20). Even for $10 t_{\text {bin }}<\tau_{\text {cut-off }}<0.1 t_{\text {tot }}$ and $\tau_{\text {cut-off }}<\tau_{\text {on }}$ the determined cutoff time can be threshold dependent (see Fig. 3(a)). An upper limit for $\tau_{\text {cut-off }}$ will then be found with the highest threshold, closest to $I_{\mathrm{on}}$. Thus, the only evidence for a true cut-off time is a threshold independence.

\section{CONCLUSION}

In summary, we test the validity of data obtained by binning and thresholding for the power law blinking model. With simulations we show that many exponents that are extracted to on- and off-state distributions in experiments cannot reflect underlying power law statistics. Only for the special case of $\alpha_{\text {off }} \approx \alpha_{\text {on }} \lesssim 1.6$ or $t_{c} \sim t_{\text {bin }}$ the method can reliably be applied independent from threshold. Otherwise, an inappropriate threshold distorts the underlying power law distribution, often beyond recognition. We were able to define three rules for most appropriate thresholding. They minimize artifacts of unresolved events independent of the actual underlying statistics. Most importantly, different thresholds should be used for on- and off-state, always close to the corresponding state, while still outside of its Poisson noise. Second, comparisons of different measurements are only meaningful, when a threshold at a similar on-state fraction is applied. This is not possible for blinking traces without a distinct on-state peak, for example, those of nanorods..$^{20}$ For those any obtained onstate distribution is highly questionable. And third, despite the seemingly better statistics, less emphasis should be laid on the shortest events, as they are most likely distorted. These findings here certainly imply the same problems for cumulative distributions ${ }^{36}$ or maximum likelihood calculations of exponents ${ }^{20,26,37}$ that are based on prior binning and thresholding. Especially for time series analysis that investigates correlations between subsequent on- and off-states, ${ }^{38,39}$ the disregard of short events naturally becomes problematic. A test for potential analysis artifacts is strongly advised. Due to the higher on-state noise, conventionally chosen thresholds are low, thus mostly suitable for the off-state, but completely inadequate for the $o n$-state. The $o n$-state cut-off additionally eliminates the unperturbed long on-events. Together with a considerable influence of real intermediate intensity levels, which can easily appear power law distributed, this renders most of the data especially on on-state power law distributions highly questionable. The central feature for blinking models, however, is the observed power law statistics for both states. If according to most observations the onset $t_{c}$ cannot be found close to $t_{\text {bin }}$, those observed statistics do not represent the underlying statistics. This would leave the real statistics to be found. Our contribution thus stresses the importance of a reliable knowledge of $t_{c}$, if the power law hypothesis is to be maintained. Possibly different methods without prior binning, like the change point analysis, ${ }^{16-18,21}$ might be more suitable for this purpose.

\section{ACKNOWLEDGMENTS}

We acknowledge financial support from the European Union, the Free State of Saxony, and the DFG Forschergruppe "From Local Constraints to Macroscopic Transport."

${ }^{1}$ F. Cichos, C. von Borczyskowski, and M. Orrit, "Power-law intermittency of single emitters," Curr. Opin. Colloid Interface Sci. 12, 272 (2007).

${ }^{2}$ P. Frantsuzov, M. Kuno, B. Jankó, and R. A. Marcus, "Universal emission intermittency in quantum dots, nanorods and nanowires," Nat. Phys. 4, 519 (2008).

${ }^{3}$ J. Zhao, G. Nair, B. R. Fisher, and M. G. Bawendi, "Challenge to the charging model of semiconductor-nanocrystal fluorescence intermittency from off-state quantum yields and multiexciton blinking," Phys. Rev. Lett. 104, 157403 (2010).

${ }^{4}$ S. Rosen, O. Schwartz, and D. Oron, "Transient fluorescence of the off state in blinking $\mathrm{CdSe} / \mathrm{CdS} / \mathrm{ZnS}$ semiconductor nanocrystals is not governed by auger recombination," Phys. Rev. Lett. 104, 157404 (2010).

${ }^{5}$ A. W. Cohn, A. M. Schimpf, C. E. Gunthardt, and D. R. Gamelin, "Sizedependent trap-assisted auger recombination in semiconductor nanocrystals," Nano Lett. 13, 1810-1815 (2013). 
${ }^{6}$ K. Shimizu, R. Neuhauser, C. Leatherdale, S. Empedocles, W. Woo, and M. Bawendi, "Blinking statistics in single semiconductor nanocrystal quantum dots," Phys. Rev. B 63, 205316 (2001).

${ }^{7}$ L. Li, G. Tian, Y. Luo, H. Brismar, and Y. Fu, "Blinking, flickering, and correlation in fluorescence of single colloidal CdSe quantum dots with different shells under different excitations," J. Phys. Chem. C 117, 4844-4851 (2013).

${ }^{8}$ C. Galland, Y. Ghosh, A. Steinbrück, M. Sykora, J. A. Hollingsworth, V. I. Klimov, and H. Htoon, "Two types of luminescence blinking revealed by spectroelectrochemistry of single quantum dots," Nature 479, 203-207 (2011).

${ }^{9}$ W. Qin and P. Guyot-Sionnest, "Evidence for the role of holes in blinking: Negative and oxidized CdSe/CdS dots," ACS Nano 6, 9125-9132 (2012).

${ }^{10}$ M. Kuno, D. P. Fromm, H. F. Hamann, A. Gallagher, and D. J. Nesbitt, "Nonexponential "blinking" kinetics of single CdSe quantum dots: A universal power law behavior," J. Chem. Phys. 112, 3117 (2000).

${ }^{11}$ R. Verberk, A. van Oijen, and M. Orrit, "Simple model for the power-law blinking of single semiconductor nanocrystals," Phys. Rev. B 66, 233202 (2002).

${ }^{12}$ J. Tang and R. A. Marcus, "Mechanisms of fluorescence blinking in semiconductor nanocrystal quantum dots," J. Chem. Phys. 123, 054704 (2005).

${ }^{13}$ M. Pelton, D. G. Grier, and P. Guyot-Sionnest, "Characterizing quantumdot blinking using noise power spectra,” Appl. Phys. Lett. 85, 819 (2004).

${ }^{14}$ M. Pelton, G. Smith, N. F. Scherer, and R. A. Marcus, "Evidence for a diffusion-controlled mechanism for fluorescence blinking of colloidal quantum dots," PNAS 104, 14249 (2007).

${ }^{15}$ J. Tang, D.-H. Lee, Y.-C. Yeh, and C.-T. Yuan, "Short-time power-law blinking statistics of single quantum dots and a test of the diffusioncontrolled electron transfer model," J. Chem. Phys. 131, 064506 (2009).

${ }^{16}$ L. P. Watkins and H. Yang, "Detection of intensity change points in time-resolved single-molecule measurements," J. Phys. Chem. B 109, 617 (2005).

${ }^{17}$ K. Zhang, H. Chang, A. Fu, A. P. Alivisatos, and H. Yang, "Continuous distribution of emission states from single CdSe/ZnS quantum dots," Nano Lett. 6, 843 (2006).

${ }^{18}$ R. Schmidt, C. Krasselt, and C. von Borczyskowski, "Change point analysis of matrix dependent photoluminescence intermittency of single $\mathrm{CdSe} / \mathrm{ZnS}$ quantum dots with intermediate intensity levels," Chem. Phys. 406, 9 (2012).

${ }^{19}$ M. Lippitz, F. Kulzer, and M. Orrit, "Statistical evaluation of single nanoobject fluorescence," ChemPhysChem 6, 770 (2005).

${ }^{20}$ C. H. Crouch, O. Sauter, X. Wu, R. Purcell, C. Querner, M. Drndic, and M. Pelton, "Facts and artifacts in the blinking statistics of semiconductor nanocrystals," Nano Lett. 10, 1692 (2010).

${ }^{21}$ T. G. Terentyeva, H. Engelkamp, A. E. Rowan, T. Komatsuzaki, J. Hofkens, C.-B. Li, and K. Blank, "Dynamic disorder in single-enzyme experiments: Facts and artifacts," ACS Nano 6, 346-354 (2012).

${ }^{22}$ P. H. Sher, J. M. Smith, P. A. Dalgarno, R. J. Warburton, X. Chen, P. J. Dobson, S. M. Daniels, N. L. Pickett, and P. O'Brien, "Power law carrier dynamics in semiconductor nanocrystals at nanosecond timescales," Appl. Phys. Lett. 92, 101111 (2008).
${ }^{23}$ F. D. Stefani, W. Knoll, M. Kreiter, X. Zhong, and M. Y. Han, "Quantification of photoinduced and spontaneous quantum-dot luminescence blinking," Phys. Rev. B 72, 125304 (2005).

${ }^{24}$ I. Chung and M. G. Bawendi, "Relationship between single quantum-dot intermittency and fluorescence intensity decays from collections of dots," Phys. Rev. B 70, 165304 (2004).

${ }^{25}$ M. Kuno, D. Fromm, S. Johnson, A. Gallagher, and D. Nesbitt, "Modeling distributed kinetics in isolated semiconductor quantum dots," Phys. Rev. B 67, 125304 (2003).

${ }^{26}$ J. P. Hoogenboom, W. K. den Otter, and H. L. Offerhaus, "Accurate and unbiased estimation of power-law exponents from single-emitter blinking data," J. Chem. Phys. 125, 204713 (2006).

${ }^{27}$ See supplementary material at http://dx.doi.org/10.1063/1.4868252 for estimations to the relative importance of unresolved events for different exponents, the analytical description of intensity histograms in the long bin time limit, as well as an estimation to the induced cut-off time by Poisson noise.

${ }^{28}$ R. T. Sibatov and V. V. Uchaikin, "Statistics of photocounts of blinking fluorescence of quantum dots," Opt. Spectrosc. 108, 761 (2010).

${ }^{29}$ G. Margolin, V. Protasenko, M. Kuno, and E. Barkai, Fractals, Diffusion, and Relaxation in Disordered Complex Systems, Advances in Chemical Physics Vol. 133, edited by W. T. Coffey and Y. P. Kalmykov (John Wiley and Sons, Inc., 2005), p. 327.

${ }^{30}$ C. Krasselt, J. Schuster, and C. von Borczyskowski, "Photoinduced hole trapping in single semiconductor quantum dots at specific sites at silicon oxide interfaces,” Phys. Chem. Chem. Phys. 13, 17084-17092 (2011).

${ }^{31}$ D. Gómez, J. Van Embden, P. Mulvaney, M. Fernée, and H. RubinszteinDunlop, "Exciton- trion transitions in single CdSe-CdS Core-Shell nanocrystals," ACS Nano 3, 2281-2287 (2009).

${ }^{32}$ N. Amecke and F. Cichos, "Intermediate intensity levels during the emission intermittency of single CdSe/ZnS quantum dots," J. Lumin. 131, 375378 (2011).

${ }^{33}$ A. Biebricher, M. Sauer, and P. Tinnefeld, "Radiative and nonradiative rate fluctuations of single colloidal semiconductor nanocrystals," J. Phys. Chem. B. 110, 5174-5178 (2006).

${ }^{34}$ W. G. J. H. M. van Sark, P. L. T. M. Frederix, A. A. Bol, H. C. Gerritsen, and A. Meijerink, "Blueing, bleaching, and blinking of single CdSe/ZnS quantum dots," ChemPhysChem 3, 871 (2002).

${ }^{35}$ F. Cichos, J. Martin, and C. von Borczyskowski, "Emission intermittency in silicon nanocrystals," Phys. Rev. B 70, 115314 (2004).

${ }^{36}$ E. A. Riley, C. M. Hess, P. J. Whitham, and P. J. Reid, "Beyond power laws: A new approach for analyzing single molecule photoluminescence intermittency," J. Chem. Phys. 136, 184508 (2012).

${ }^{37}$ M. L. Goldstein, S. A. Morris, and G. G. Yen, "Problems with fitting to the power-law distribution,” Eur. Phys. J. B 41, 255 (2004).

${ }^{38}$ F. D. Stefani, X. Zhong, W. Knoll, M. Han, and M. Kreiter, "Memory in quantum-dot photoluminescence blinking," New J. Phys. 7, 197-197 (2005).

${ }^{39}$ A. A. Cordones, K. L. Knappenberger, and S. R. Leone, "Linking on-state memory and distributed kinetics in single nanocrystal blinking," J. Phys. Chem. B 117, 4241 (2013). 